\title{
Synthesis and In Vitro Antioxidant Evaluation of Some Indole-2-carboxylic Acid-Aryl Amine Conjugates
}

\author{
NAGARAJA NAIK ${ }^{\mathrm{a}^{*}}$, V. SHARATH ${ }^{\mathrm{a}}$, H. VIJAY KUMAR ${ }^{\mathrm{b}}$ and H. SWETHA ${ }^{\mathrm{c}}$ \\ ${ }^{a}$ Department of Studies in Chemistry, University of Mysore, Manasagangotri, \\ Mysore-570006, Karnataka, India \\ ${ }^{\mathrm{b}}$ Department of Organic Chemistry, Indian Institute of Science, \\ Banglore-570012, Karnataka, India \\ ${ }^{\mathrm{c}}$ Plantation Products, Spices \& Flavour Technology, \\ Central Food Technological and Research Institute, Mysore-570006, Karnataka, India \\ drnaikchem@gmail.com
}

Received 29 July 2012 / Accepted 4 September 2012

\begin{abstract}
A simple and efficient protocol for the synthesis of novel indole-2-carboxylic acid analogues having aryl amine moieties (2a-k) has been described. The synthesized compounds were characterized by spectroscopic techniques (IR, ${ }^{1} \mathrm{H}$ NMR, ${ }^{13} \mathrm{C}$ NMR and MS-EI) and further screened for their antioxidant activity by using various in vitro assays such as 2,2-diphenyl-1-picrylhydrazyl $\left(\mathrm{DPPH}^{*}\right)$ scavenging assay, 2,2'-azino-bis(3-ethylbenzthiazoline-6-sulfonic acid) (ABTS ${ }^{-+}$) radical scavenging activity, ferric reducing antioxidant power, cupric ion reducing ability method and phosphomolybdate method. In all the antioxidant assays performed, compounds $\mathbf{2 b}, \mathbf{2 d}, \mathbf{2 e}$ and $\mathbf{2 f}$ showed promising antioxidant activity. Whereas, compound 2c displayed potent antioxidant activity.
\end{abstract}

Keywords: Free radicals, Antioxidant activity, Indole-2-carboxylic acid, Aryl amines

\section{Introduction}

Reactive oxygen species (ROS) and free radicals have attracted increasing attention over the past decade. Free radicals are exacerbating factors in cellular injury and aging process ${ }^{1-3}$. Antioxidants, used to prevent or inhibit the natural phenomena of oxidation, have a broad application in diverse industrial fields as they have a huge importance either as industrial additives or health agents ${ }^{4,5}$. Research data have revealed that they could be suitable for preventive and therauptic purposes in several diseases related with oxidative stress (e.g. atherosclerosis, inflammatory injury, cancer and cardiovascular diseases) ${ }^{6,7}$. In the vast heterocyclic structural space, the indole nucleus occupies a position of major importance. Many indole derivatives including fused derivatives form the basis of a range of pharmaceuticals ${ }^{8,9}$.

Indole moiety occurs widely in synthetic and natural products containing an important class of therapeutic agents ${ }^{10,11}$. In the last decade, antioxidant activity of synthetic indole 
derivatives and their possible activity mechanisms have been widely studied ${ }^{11}$. Generally, pharmacophores containing typically the phenol moiety possess good antioxidant activity $^{12}$. In addition to the traditional $\mathrm{O}-\mathrm{H}$ bond type antioxidant, tricyclic amines having $\mathrm{N}-\mathrm{H}$ bond functions as the antioxidant have attracted much research attention because aromatic amines $\left(\mathrm{Ar}_{2} \mathrm{NH}\right)$ have always been the central structure in many currently used drugs ${ }^{13}$. It was envisaged, that the two pharmacophores if linked together would generate novel molecular templates, which are likely to exhibit interesting antioxidant properties. Owing to the importance and in continuation of our work on synthesis of novel biologically active heterocyclic compounds ${ }^{14-17}$, in the present investigation we have focused on the synthesis and evaluation of antioxidant activity of novel indole-2-carboxylic acid analogues. Our study reveals that assembling of indole-2carboxylic acid with aryl amine moiety dispatch the generous support for the enhancement in the antioxidant activity.

\section{Experimental}

All the chemicals and reagents were purchased commercially from Merck, Himedia and SD Fine Chemicals and were used without further purification. Melting points of the synthesized compounds were determined in open capillaries and are uncorrected. Reactions are monitored by thin-layer chromatography (TLC) on silica gel $60 \mathrm{~F}_{254}$ aluminum sheets (Merck). IR spectra are recorded in $\mathrm{KBr}$ on FT-IR021 spectrophotometer $\left(v_{\max }\right.$ in $\left.\mathrm{cm}^{-1}\right)$. ${ }^{1} \mathrm{H}$ NMR and ${ }^{13} \mathrm{C}$ NMR on Jeol GSX $400-\mathrm{MHz}$ spectrometer (chemical shift in $\delta \mathrm{ppm}$ downfield from TMS as an internal reference). The mass spectra were recorded on WatersQ-TOF Ultima spectrometer. The elemental analysis was determined on FLASH EA-1112 series instrument. All the compounds gave $\mathrm{C}, \mathrm{H}, \mathrm{O}$ and $\mathrm{N}$ analysis within $\pm 0.4 \%$ of the theoretical values.

\section{Chemistry}

$\mathrm{N}$-Acylation of indole-2-carboxylic acid with 2-chloroacetylchloride in the presence of triethylamine (TEA) as a base yields the key scaffold 1-(2-chloroacetyl)-1H-indole-2carboxylic acid (2) Scheme 1. Further, coupling of various aryl amines to key scaffold (2) by base condensation reaction affords novel indole-2-carboxylic acid analogues (2a-k) in moderate to good yield, which were identified by spectroscopic techniques: NMR $\left({ }^{1} \mathrm{H}\right.$ NMR, ${ }^{13} \mathrm{C}$ NMR), FT-IR and MS-EI. A typical synthetic strategy employed to obtain the title compound (2a-k) is depicted in Scheme 2.

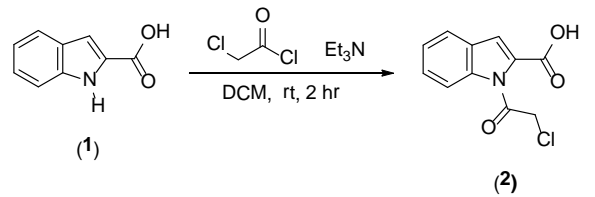

Scheme 1. Reaction for the synthesis of indole-2-carboxylic acid-aryl amine conjugates (2a-k)

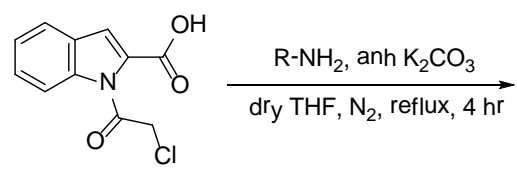

(2)<smiles>[R]NCC(=O)n1c(C(=O)O)cc2ccccc21</smiles>

$(2 \mathrm{a}-\mathbf{k})$

Scheme 2. Pathway for the synthesis of indole-2-carboxylic acid-aryl amine conjugates (2a-k) 
Procedure for the synthesis of 1-(2-chloroacetyl)-1H-indole-2-carboxylic acid (compound 2)

To a well stirred solution of indole-2-carboxylic acid (1 mM) and triethylamine (1.2 $\mathrm{mM})$ in $10 \mathrm{~mL}$ dichloromethane, 2-chloroacetylchloride $(1.2 \mathrm{mM})$ in $5 \mathrm{ml}$ was added drop by drop for $10 \mathrm{~min}$, then the reaction mixture was stirred at room temperature for about $2 \mathrm{~h}$. Progress of the reaction was monitored by TLC using hexane:ethyl acetate (6:4) mixture as mobile phase. After the completion of reaction, the reaction mass was quenched in ice cold water and the product was extracted with diethyl ether. The organic layer was washed with brine solution followed by distilled water and dried briefly $\left(\mathrm{Na}_{2} \mathrm{SO}_{4}\right)$ and the volatiles were removed in vacuo at $<30{ }^{\circ} \mathrm{C}$, the resulting brownish solid was identified.

Brownish solid, yield (85\%), m.p. 185-188 ${ }^{\circ} \mathrm{C}$, IR (KBr) $v_{\max }\left(\mathrm{cm}^{-1}\right)$ : $3349.75(\mathrm{COOH})$, 2926.60 (Ar-H), $1603.2(\mathrm{C}=\mathrm{O}) ;{ }^{1} \mathrm{H}$ NMR $\left.(400 \mathrm{MHz})\left(\mathrm{CDCl}_{3}\right) \delta \mathrm{ppm}\right): 11.0$ (s, $\left.1 \mathrm{H}, \mathrm{COOH}\right)$, 7.33 (s, $1 \mathrm{H}, \mathrm{CH}$ of indole), 6.87-8.11 (m, 4H, Ar-H), 4.49 (s, $2 \mathrm{H}, \mathrm{CH}_{2} \mathrm{Cl}$ ); Mass $(\mathrm{m} / \mathrm{z}): \mathrm{M}^{+}$ 238.02; Anal. Calcd. for $\mathrm{C}_{11} \mathrm{H}_{8} \mathrm{ClNO}_{3}$ : C, 55.60; H, 3.39; N, 5.89; O, 20.20\%; Found; C, 55.57; H, 3.43; N, 5.85; O, 20.24\%.

General procedure for the synthesis for the indole-2-carboxylic acid conjugated with aryl amine analogues (2a-k)

A mixture of aryl amines $(1.2 \mathrm{mM})$ and $\mathrm{K}_{2} \mathrm{CO}_{3}(1 \mathrm{mM})$ in dry tetrahydrofuran (dry THF, $10 \mathrm{~mL}$ ) were stirred under $\mathrm{N}_{2}$ atmosphere for $30 \mathrm{~min}$ at room temperature. The mixture was treated drop wise with 1-(2-chloroacetyl)- $1 H$-indole-2-carboxylic acid $(1 \mathrm{mM})$ in dry THF $(10 \mathrm{~mL})$ and refluxed for $4 \mathrm{~h}$. The progress of the reaction was monitored by TLC. The reaction mixture was then desolventized in rotary evaporator and the product was extracted in ethyl acetate and washed with water and dried over anhydrous $\mathrm{Na}_{2} \mathrm{SO}_{4}$. The products were separated and purified by column chromatography, using hexane:ethyl acetate=60:40.

\section{1-(2-(Phenylamino)acetyl)-1H-indole-2-carboxylic acid (compound 2a)}

Brown solid, m.p. 137-139 ${ }^{\circ} \mathrm{C}$, IR (KBr) $v_{\max }\left(\mathrm{cm}^{-1}\right)$ : $3623.32(\mathrm{COOH}), 3330.46(\mathrm{~N}-\mathrm{H})$, 2854.17-2938.52 (Ar-H),1653.66 (C=O ); ${ }^{1} \mathrm{H}$ NMR (400 MHz) $\left(\mathrm{CDCl}_{3}\right)(\delta \mathrm{ppm}): 11.0$ (s, $1 \mathrm{H}, \mathrm{COOH}), 7.1-8.11$ (m, 9H, Ar-H), 7.33 (s, $1 \mathrm{H}, \mathrm{CH}$ of indole), $4.17\left(\mathrm{~s}, 2 \mathrm{H}, \mathrm{CH}_{2} \mathrm{~N}-\mathrm{H}\right)$, 4.0 (s, $1 \mathrm{H}, \mathrm{NH}) ;{ }^{13} \mathrm{C} \mathrm{HMR}\left(\mathrm{CDCl}_{3}\right)(\delta \mathrm{ppm}): 168.2,160.1,147.5,141.1,129.5,127.4,124.4$, 123.5, 120.8, 115.6, 53.1; Mass (m/z): $\mathrm{M}^{+}$295.3; Anal. Calcd. for $\mathrm{C}_{17} \mathrm{H}_{14} \mathrm{~N}_{2} \mathrm{O}_{3}$ : C, 69.38; $\mathrm{H}$, 4.79; N, 9.52; O, 16.31\%; Found; C, 69.40; H, 4.81; N, 9.53; O, 16.30\%.

\section{1-(2-(2-Hydroxyphenylamino)acetyl)-1H-indole-2-carboxylic acid (compound $2 \boldsymbol{b}$ )}

Light yellow solid, m.p. 157-159 ${ }^{\circ} \mathrm{C}$, IR $(\mathrm{KBr}) v_{\max }\left(\mathrm{cm}^{-1}\right)$ : $3344.16(\mathrm{COOH}), 3316.12(\mathrm{~N}-\mathrm{H})$, 2853.51-2930.85 (Ar-H), $1605.40(\mathrm{C}=\mathrm{O}) ;{ }^{1} \mathrm{H}$ NMR (400 MHz) $\left(\mathrm{CDCl}_{3}\right)(\delta \mathrm{ppm}): 11.2(\mathrm{~s}, 1 \mathrm{H}$, $\mathrm{COOH}$ ), 7.23 (s, $1 \mathrm{H}, \mathrm{CH}$ of indole), 6.83-8.20 (m, 8H, Ar-H), 5.35 (s, $1 \mathrm{H}, \mathrm{OH}$ of Phenol), 4.3 (s, $1 \mathrm{H}, \mathrm{NH}), 4.15$ (s, $\left.2 \mathrm{H}, \mathrm{CH}_{2} \mathrm{~N}-\mathrm{H}\right) ;{ }^{13} \mathrm{C} \mathrm{HMR}\left(\mathrm{CDCl}_{3}\right)(\delta \mathrm{ppm}): 168.3,160.1,141.7,140.9,139.8$, 127.3, 124.4, 123.2, 120.0, 118.6, 114.8, 53.3; Mass (m/z): $\mathrm{M}^{+}$311.50; Anal. Calcd. for $\mathrm{C}_{17} \mathrm{H}_{14} \mathrm{~N}_{2} \mathrm{O}_{4}$ : C, 65.80; H, 4.55; N, 9.03; O, 20.62\%; Found; C, 65.83; H, 4.52; N, 9.07; O, 20.60\%.

\section{1-(2-(4-Hydroxyphenylamino)acetyl)-1H-indole-2-carboxylic acid (compound 2c)}

Yellow solid, m.p. $153-155{ }^{\circ} \mathrm{C}, \mathrm{IR}(\mathrm{KBr}) v_{\max }\left(\mathrm{cm}^{-1}\right): 3622.63(\mathrm{COOH}), 3306.46(\mathrm{~N}-\mathrm{H})$, 2853.58-2923.56 (Ar-H), $1689.98(\mathrm{C}=\mathrm{O})$ ); ${ }^{1} \mathrm{H}$ NMR (400 MHz) $\left(\mathrm{CDCl}_{3}\right)(\delta \mathrm{ppm}: 11.0$ (s, 1H, COOH), 7.4 (s, 1H, CH of indole), 6.92-8.11 (m, 8H, Ar-H), 5.42 (s, 1H, OH), 4.17 (s, $\left.2 \mathrm{H}, \mathrm{CH}_{2} \mathrm{~N}-\mathrm{H}\right)$,), 4.0 (s, $\left.1 \mathrm{H}, \mathrm{NH}\right) ;{ }^{13} \mathrm{C} \mathrm{HMR}\left(\mathrm{CDCl}_{3}\right)(\delta \mathrm{ppm}): 168.4,160.2,146.8,141.1$, 
127.5, 126.6, 124.3, 119.5, 116.8, 115.8, 53.2; Mass (m/z): $\mathrm{M}^{+}$311.13; Anal. Calcd. for $\mathrm{C}_{17} \mathrm{H}_{14} \mathrm{~N}_{2} \mathrm{O}_{4}$ : C, 65.80; H, 4.55; N, 9.03; O, 20.62\%; Found; C, 65.83; H, 4.52; N, 9.06; O, $20.60 \%$.

\section{1-(2-(2-Methoxyphenylamino)acetyl)-1H-indole-2-carboxylic acid (compound 2d)}

Pale brown solid, m.p. 160-162 ${ }^{\circ} \mathrm{C}$, IR (KBr) $v_{\max }\left(\mathrm{cm}^{-1}\right)$ : $3394.32(\mathrm{COOH}), 3334.32(\mathrm{~N}-\mathrm{H})$, 2862.17-2938.52 (Ar-H), $1615.52(\mathrm{C}=\mathrm{O}) ;{ }^{1} \mathrm{H}$ NMR $(400 \mathrm{MHz})\left(\mathrm{CDCl}_{3}\right)(\delta \mathrm{ppm}): 11.3(\mathrm{~s}, 1 \mathrm{H}$, $\mathrm{COOH}$ ), 7.35 (s, $1 \mathrm{H}, \mathrm{CH}$ of indole), 6.86-8.21 (m, 8H, Ar-H), 4.19 (s, $\left.2 \mathrm{H}, \mathrm{CH}_{2} \mathrm{~N}-\mathrm{H}\right), 4.0$ (s, $1 \mathrm{H}$, $\mathrm{NH}), 3.83$ (s, 3H, $\left.\mathrm{OCH}_{3}\right) ;{ }^{13} \mathrm{C} \mathrm{HMR}\left(\mathrm{CDCl}_{3}\right)(\delta \mathrm{ppm}): 168.3,160.1,144.6,141.1,128.3,127.4$, 124.4, 123.6, 121.8, 119.6, 55.9, 53.1; Mass (m/z): $\mathrm{M}^{+}$325.01; Anal. Calcd. for $\mathrm{C}_{18} \mathrm{H}_{16} \mathrm{~N}_{2} \mathrm{O}_{4}$ : C, 66.66; H, 4.97; N, 8.64; O, 19.73\%; Found; C, 66.68; H, 4.96; N, 8.61; O, 19.70\%.

\section{1-(2-(4-Methoxyphenylamino)acetyl)-1H-indole-2-carboxylic acid (compound 2e)}

Pale brown semisolid, IR (KBr) $v_{\max }\left(\mathrm{cm}^{-1}\right)$ : $3589.51(\mathrm{COOH}), 3316.16(\mathrm{~N}-\mathrm{H}), 2852.54-$ 2924.52 (Ar-H), 1630.88 (C=O ); ${ }^{1} \mathrm{H}$ NMR (400 MHz) $\left.\left(\mathrm{CDCl}_{3}\right) \delta \mathrm{ppm}\right): 11.0$ (s, $\left.1 \mathrm{H}, \mathrm{COOH}\right)$, 7.42 (s, $1 \mathrm{H}, \mathrm{CH}$ of indole), 6.87-8.11(m, 8H, Ar-H), 4.27 (s, $\left.2 \mathrm{H}, \mathrm{CH}_{2} \mathrm{~N}-\mathrm{H}\right), 4.1$ (s, $\left.1 \mathrm{H}, \mathrm{NH}\right)$, $3.86\left(\mathrm{~s}, 3 \mathrm{H}, \mathrm{OCH}_{3}\right) ;{ }^{13} \mathrm{C}$ HMR $\left(\mathrm{CDCl}_{3}\right)(\delta \mathrm{ppm}): 168.2,160.3,151.6,141.2,139.6,127.4$, 124.2, 123.5, 119.9, 55.9, 53.1; Mass (m/z): $\mathrm{M}^{+}$325.13; Anal. Calcd. for $\mathrm{C}_{18} \mathrm{H}_{16} \mathrm{~N}_{2} \mathrm{O}_{4}$ : C, 66.66; H, 4.97; N, 8.64; O, 19.73\%; Found; C, 66.65; H, 4.99; N, 8.62; O, 19.71\%.

1-(2-(2,4-Dimethoxyphenylamino)acetyl)-1H-indole-2-carboxylic acid (compound 2f) Yellow semisolid, IR (KBr) $v_{\max }\left(\mathrm{cm}^{-1}\right)$ : $3626.48(\mathrm{COOH}), 3334.46(\mathrm{~N}-\mathrm{H}), 2853.17-2924.52$ (Ar-H), 1687.66 (C=O ); ${ }^{1} \mathrm{H}$ NMR (400 MHz) $\left.\left(\mathrm{CDCl}_{3}\right) \delta \mathrm{ppm}\right): 11.0$ (s, 1H, COOH), 7.858.11 (m, 8H, Ar-H), 7.33 (s, 1H, CH of indole), 4.19 (s, $1 \mathrm{H}, \mathrm{NH}), 4.12$ (s, $\left.2 \mathrm{H}, \mathrm{CH}_{2} \mathrm{~N}-\mathrm{H}\right), 3.87$ (s, $\left.6 \mathrm{H}, \mathrm{OCH}_{3}\right) ;{ }^{13} \mathrm{C}$ HMR $\left(\mathrm{CDCl}_{3}\right)(\delta \mathrm{ppm}): 168.4,162.3,160.3,145.6,141.1,130.7,126.4$, 124.1, 119.8, 101.3, 55.8, 53.2; Mass (m/z): $\mathrm{M}^{+}$355.26; Anal. Calcd. for $\mathrm{C}_{19} \mathrm{H}_{18} \mathrm{~N}_{2} \mathrm{O}_{5}$ : C, 64.40; H, 5.12; N, 7.91; O, 22.58\%; Found; C, 64.43; H, 5.13; N, 7.95; O, 22.54\%.

\section{1-(2-(p-Tolylamino)acetyl)-1H-indole-2-carboxylic acid (compound 2g)}

White solid, m.p. $167-169{ }^{\circ} \mathrm{C}$, IR (KBr) $v_{\max }\left(\mathrm{cm}^{-1}\right)$ : $3621.66(\mathrm{COOH}), 3340.16(\mathrm{~N}-\mathrm{H})$, 2854.13-2954.82 (Ar-H), 1618.95 (C=O ); ${ }^{1} \mathrm{H}$ NMR (400 MHz) $\left.\left(\mathrm{CDCl}_{3}\right) \delta \mathrm{ppm}\right): 11.6(\mathrm{~s}, 1 \mathrm{H}$, $\mathrm{COOH}$ ), 7.13 (s, $1 \mathrm{H}, \mathrm{CH}$ of indole), 7.0-8.11(m, 8H, Ar-H), 4.27 (s, $\left.2 \mathrm{H}, \mathrm{CH}_{2} \mathrm{~N}-\mathrm{H}\right), 4.1$ (s, $1 \mathrm{H}$, $\mathrm{NH}), 2.32$ (s, $\left.3 \mathrm{H}, \mathrm{CH}_{3}\right) ;{ }^{13} \mathrm{C} \mathrm{HMR}\left(\mathrm{CDCl}_{3}\right)(\delta \mathrm{ppm}): 168.3,160.2,144.5,141.2,130.7,126.8$, 124.3, 119.8, 113.2, 53.6, 21.4; Mass (m/z): $\mathrm{M}^{+}$309.06; Anal. Calcd. for $\mathrm{C}_{18} \mathrm{H}_{16} \mathrm{~N}_{2} \mathrm{O}_{3}$ : C, 70.12; H, 5.23; N, 9.09; O, 15.57\%; Found; C, 70.15; H, 5.26; N, 9.07; O, 15.55\%.

\section{1-(2-(4-Chlorophenylamino)acetyl)-1H-indole-2-carboxylic acid (compound $\mathbf{2 h}$ )}

Light brown solid, m.p. $143-145^{\circ} \mathrm{C}$, IR (KBr) $v_{\max }\left(\mathrm{cm}^{-1}\right)$ : $3538.31(\mathrm{COOH}), 3309.16(\mathrm{~N}-\mathrm{H})$, 2853.17-2935.52 (Ar-H),1620.88 (C=O); ${ }^{1} \mathrm{H}$ NMR (400 MHz) $\left.\left(\mathrm{CDCl}_{3}\right) \delta \mathrm{ppm}\right): 11.1(\mathrm{~s}, 1 \mathrm{H}$, $\mathrm{COOH}), 6.87-8.11(\mathrm{~m}, 8 \mathrm{H}, \mathrm{Ar}-\mathrm{H}), 7.33$ (s, $1 \mathrm{H}, \mathrm{CH}$ of indole), 4.18 (s, 2H, $\left.\mathrm{CH}_{2} \mathrm{~N}-\mathrm{H}\right), 4.1$ (s, $1 \mathrm{H}, \mathrm{NH}) ;{ }^{13} \mathrm{C} \mathrm{HMR}\left(\mathrm{CDCl}_{3}\right)(\delta \mathrm{ppm}): 168.3,145.7,141.6,129.6,126.0,124.2,123.4$, 119.7, 114.8, 53.2; Mass (m/z): $\mathrm{M}^{+}$330.08; Anal. Calcd. for $\mathrm{C}_{17} \mathrm{H}_{13} \mathrm{ClN}_{2} \mathrm{O}_{3}$ : C, 62.11; $\mathrm{H}$, 3.99N, 8.52; O, 14.60\%; Found; C, 62.13; H, 3.97; N, 8.56; O, 14.61\%.

\section{1-(2-(4-Bromophenylamino)acetyl)-1H-indole-2-carboxylic acid (compound 2i)}

Brown solid, m.p. 138-140 ${ }^{\circ} \mathrm{C}$, IR (KBr) $v_{\max }\left(\mathrm{cm}^{-1}\right): 3438.31(\mathrm{COOH}), 3312.96(\mathrm{~N}-\mathrm{H})$, 2848.17-2924.52 (Ar-H), 1618.95 (C=O ); ${ }^{1} \mathrm{H}$ NMR (400 MHz) $\left.\left(\mathrm{CDCl}_{3}\right) \delta \mathrm{ppm}\right): 11.2(\mathrm{~s}, 1 \mathrm{H}$, 
$\mathrm{COOH}$ ), 6.87-8.11 (m, 8H, Ar-H), 7.31 (s, 1H, CH of indole), 4.18 (s, 2H, $\left.\mathrm{CH}_{2} \mathrm{~N}-\mathrm{H}\right), 4.0$ (s, $1 \mathrm{H}, \mathrm{NH}) ;{ }^{13} \mathrm{C} \mathrm{HMR}\left(\mathrm{CDCl}_{3}\right)(\delta \mathrm{ppm}): 168.2,160.2,146.5,132.3,126.4,124.3,119.7$, 115.0, 114.2, 53.1; Mass (m/z): $\mathrm{M}^{+}$373.06; Anal. Calcd. for $\mathrm{C}_{17} \mathrm{H}_{13} \mathrm{BrN}_{2} \mathrm{O}_{3}$ : C, 54.71; $\mathrm{H}$, 3.51; N, 7.51; O, 12.86\%; Found; C, 54.73; H, 3.54; N, 7.48; O, 12.87\%.

\section{1-(2-(2-Nhitrophenylamino)acetyl)-1H-indole-2-carboxylic acid (compound 2j)}

Pale yellow solid, m.p. 118-120 ${ }^{\circ} \mathrm{C}$, IR (KB) $v_{\max }\left(\mathrm{cm}^{-1}\right)$ : $3621.66(\mathrm{COOH}), 3353.60(\mathrm{~N}-\mathrm{H})$, 2854.13-2923.56 (Ar-H), $1625.70(\mathrm{C}=\mathrm{O}) ;{ }^{1} \mathrm{H}$ NMR $\left.(400 \mathrm{MHz})\left(\mathrm{CDCl}_{3}\right) \delta \mathrm{ppm}\right): 11.6$ (s, $1 \mathrm{H}, \mathrm{COOH}), 6.87-8.11$ (m, 8H, Ar-H), 7.31 (s, $1 \mathrm{H}, \mathrm{CH}$ of indole), 4.17 (s, $\left.2 \mathrm{H}, \mathrm{CH}_{2} \mathrm{~N}-\mathrm{H}\right)$, 4.0 (s, $1 \mathrm{H}, \mathrm{NH}) ;{ }^{13} \mathrm{C} \mathrm{HMR}\left(\mathrm{CDCl}_{3}\right)(\delta \mathrm{ppm}): 168.2,160.1,145.4,131.7,135.4,125.6,124.2$, 123.5, 119.8, 108.6, 52.2; Mass (m/z): $\mathrm{M}^{+}$340.05; Anal. Calcd. for $\mathrm{C}_{17} \mathrm{H}_{13} \mathrm{~N}_{3} \mathrm{O}_{5}$ : C, 60.18; H, 3.86; N, 12.38; O, 23.58\%; Found; C, 60.16; H, 3.85; N, 12.41; O, 23.59\%.

\section{1-(2-(4-Nitrophenylamino)acetyl)-1H-indole-2-carboxylic acid (compound $\mathbf{2 k}$ )}

Pale yellow solid, m.p. $125-128{ }^{\circ} \mathrm{C}$, IR (KBr) $v_{\max }\left(\mathrm{cm}^{-1}\right)$ : $3478.95(\mathrm{COOH}), 3358.43(\mathrm{~N}-\mathrm{H})$, 2853.17-2924.52 (Ar-H), $1630.52(\mathrm{C}=\mathrm{O}) ;{ }^{1} \mathrm{H}$ NMR (400 MHz) $\left.\left(\mathrm{CDCl}_{3}\right) \delta \mathrm{ppm}\right): 11.0$ (s, $1 \mathrm{H}, \mathrm{COOH}), 6.87-8.20$ (m, 8H, Ar-H),), 7.30 (s, $1 \mathrm{H}, \mathrm{CH}$ of indole), 4.17 (s, $2 \mathrm{H}, \mathrm{CH}_{2} \mathrm{~N}-\mathrm{H}$ 4.0 (s, $1 \mathrm{H}, \mathrm{NH}) ;{ }^{13} \mathrm{C} \mathrm{HMR}\left(\mathrm{CDCl}_{3}\right)(\delta \mathrm{ppm}): 168.4,160.3,153.8,141.2,136.2,127.7,126.5$, 123.5, 119.8, 114.3, 108.4, 53.1; Mass (m/z): $\mathrm{M}^{+}$341.20; Anal. Calcd. for $\mathrm{C}_{17} \mathrm{H}_{13} \mathrm{~N}_{3} \mathrm{O}_{5}$ : C, 60.18; H, 3.86; N, 12.38; O, 23.58\%; Found; C, 60.19; H, 3.84; N, 12.36; O, 23.59\%.

\section{Results and Discussion}

A series of novel indole-2-carboxylic acid derivatives (2a-k) were synthesized. Scheme 1 and Scheme 2 illustrates the way used for the preparation of target molecules. The starting material, indole-2-carboxylic acid (1) reacts with chloroacetyl chloride in the presence of triethylamine as a base to yield 1-(2-chloroacetyl)- $1 H$-indole-2-carboxylic acid (2), a key scaffold Scheme 1. Further, in order to assemble the key scaffold (2) and different aryl amines and to study the effect on antioxidant properties, the key scaffold (2) was conjugated with different aryl amines in the presence of potassium carbonate as a base to afford desired products (2a-k) Scheme 2. Structural conformation was done using IR, ${ }^{1} \mathrm{H}$ $\mathrm{NMR},{ }^{13} \mathrm{C}$ NMR, mass spectra and elemental analysis. In the IR spectrum of 1-(2chloroacetyl)- $1 \mathrm{H}$-indole-2-carboxylic acid (2) the $\mathrm{C}=\mathrm{O}$ stretching band was observed at $1603.2 \mathrm{~cm}^{-1}$ and absence of N-H band at $3310 \mathrm{~cm}^{-1}$ confirms the $N$-acylation reaction. The structure of the compound (2) was further confirmed by ${ }^{1} \mathrm{H}$ NMR spectrum where the absence of the signal at $\delta 11.1 \mathrm{ppm}$ which corresponds to $\mathrm{N}-\mathrm{H}$ proton of indole ring. All the respective aromatic protons were signaled at $\delta 6.87-8.11 \mathrm{ppm} .{ }^{1} \mathrm{H}$ NMR spectra of all indole-2-carboxylic acid aryl-amine conjugates (2a-k) showed multiplet for Ar-H proton at $\delta 6.76-8.11 \mathrm{ppm}$ and singlet for $\mathrm{N}-\mathrm{H}$ protons at $\delta 4.0-4.1 \mathrm{ppm}$. All the analogues showed mass according to their $\mathrm{M}^{+}$ions.

\section{Antioxidant activities}

All the newly synthesized series of indole-2-carboxylic acid conjugated aryl-amine derivatives (2a-k) were screened for their antioxidant potential by employing different in vitro assays such as, DPPH free radical scavenging assay and 2,2'-azino-bis(3ethylbenzthiazoline-6-sulfonic acid) $\left(\mathrm{ABTS}^{*+}\right.$ ) radical scavenging activity, ferric reducing antioxidant power, cupric ion reducing ability method and phosphomolybdate method. All the in vitro assay measurements were performed in triplicates. 


\section{$D P P H$ free radical scavenging activity}

The newly synthesized compounds were screened for free radical scavenging activity by DPPH method ${ }^{18}$. DPPH is a stable nitrogen-centered free radical, the color of which changes from violet to yellow upon reduction by either the process of hydrogen or electron donation. The samples and the standards like butylated hydroxy anisole (BHA) and ascorbic acid were prepared at concentrations of 10, 25, 50, 100, 200 and $500 \mu \mathrm{M}$. Among the tested indole-2carboxylic acid conjugated with aryl amine analogues (2a-k) displayed certain degree of activity. The results of the analogues appeared to be related to the nature of the substituent group on the substituted phenyl ring of aryl amines. Among the synthesized analogues, compound 2c exhibited better scavenging activity (Table 2), this may be due to the presence of hydroxyl group on the para substituted phenyl ring. Chloro, bromo and nitro substituted indole-2-carboxylic acid derivatives (2h-k) showed least scavenging activities compared to standard.

Table 1. Chemical structures and yields of indole-2-carboxylic acid aryl-amine conjugates

Compound


Table 2. Concentration required for $50 \%$ scavenging $\left(\mathrm{IC}_{50}\right)$ of $\mathrm{DPPH}^{*}$ and $\mathrm{ABTS}^{*+}$ radical scavenging activities of the compounds (2a-k) and the standard antioxidant compounds BHA and Ascorbic acid. Each value represents mean \pm SD $(n=3)$

\begin{tabular}{ccc}
\hline \multirow{2}{*}{ Tested compounds } & \multicolumn{2}{c}{ Scavenging activity $\left(\mathrm{IC}_{50}\right)^{\mathrm{a}}$} \\
\cline { 2 - 3 } & $\mathrm{DPPH}^{\bullet}$ & ABTS $^{+}$ \\
\hline BHA & $12.2 \pm 0.12$ & $20.9 \pm 0.12$ \\
Ascorbic acid & $11.1 \pm 0.11$ & $18.3 \pm 0.14$ \\
01 & $124 \pm 0.34$ & $88.4 \pm 0.16$ \\
$\mathbf{2 a}$ & $156 \pm 0.21$ & $132.1 \pm 0.35$ \\
$\mathbf{2 b}$ & $18 \pm 0.25$ & $36.6 \pm 0.24$ \\
$\mathbf{2} \mathbf{c}$ & $15 \pm 0.63$ & $29.3 \pm 0.45$ \\
$\mathbf{2 d}$ & $81 \pm 0.62$ & $153.16 \pm 0.81$ \\
$\mathbf{2}$ & $61 \pm 0.54$ & $132.62 \pm 0.35$ \\
$\mathbf{2 f}$ & $26 \pm 0.20$ & $32.7 \pm 0.12$ \\
$\mathbf{2 g}$ & $132 \pm 0.51$ & $189.03 \pm 0.33$ \\
$\mathbf{2 h}$ & $189 \pm 0.11$ & $173.12 \pm 0.10$ \\
$\mathbf{2} \mathbf{i}$ & $301 \pm 0.72$ & $350.85 \pm 0.53$ \\
$\mathbf{2 j}$ & $234 \pm 0.45$ & $203.34 \pm 0.60$ \\
$\mathbf{2}$ & $261 \pm 0.16$ & $293.32 \pm 0.27$ \\
\hline
\end{tabular}

${ }^{a}$ The values are expressed as $\mu M$ concentration. Lower $I C_{50}$ values indicate higher radical scavenging activity

\section{$\mathrm{ABTS}^{\circ+}$ radical scavenging activity}

The synthesized indole-2-carboxylic acid aryl-amine conjugates were subjected to ABTS ${ }^{*+}$ radical scavenging activity ${ }^{19}$. This assay is based on a decolorization technique in which the radical is generated directly in a stable form prior to reaction with putative antioxidants. In this assay, the technique is based on the direct production of the blue/green ABTS $^{*+}$ chromophore through the reaction between ABTS and potassium persulfate. Amongst the synthesized analogues, compounds 2c, $2 \mathbf{b}$ and $2 \mathbf{2 f}$ showed promising $\mathrm{ABTS}^{*+}$ scavenging activity and compounds $\mathbf{2 g}$, $\mathbf{2 e}$ and $\mathbf{2 d}$ displayed moderate scavenging activity. Whereas, compounds $2 \mathbf{h}, \mathbf{2} \mathbf{i}, \mathbf{2} \mathbf{j}$ and $2 \mathbf{k}$ possessing chloro and nitro substituents showed very least $\mathrm{ABTS}^{*+}$ scavenging activity compared to standard BHA and ascorbic acid (Table 2).

\section{Ferric reducing antioxidant power (FRAP) assay}

All the synthesized indole-2-carboxylic acid aryl-amine conjugates were screened for ferric reducing antioxidant power ${ }^{20}$. The FRAP method is based on the reduction of $\mathrm{Fe}^{3+}-\mathrm{Fe}^{2+}$. The presence of antioxidants in the samples would result in the reducing $\mathrm{Fe}^{3+}$ to $\mathrm{Fe}^{2+}$ by donating an electron. The amount of $\mathrm{Fe}^{2+}$ complex can be then monitored by measuring the formation of Prussian blue at $700 \mathrm{~nm}$. The reducing ability of the compound may serve as a significant indicator of its potential antioxidant activity. Compounds $\mathbf{2 b}, \mathbf{2 f}, \mathbf{2 d}$ and $2 \mathbf{2 e}$ displayed good ferric ion reducing ability compared to other scaffolds. Compound $\mathbf{2 c}$ has the most powerful ferric ion reducing ability, this may be due to the electron donating power of hydroxy group present in the substituted phenyl ring. Also the results from the (Table 3) reveals that the presence of electron withdrawing groups on the substituted phenyl ring did not favors in the ferric ion reducing ability. 
Table 3. Comparison of ferric ions $\left(\mathrm{Fe}^{3+}\right)$ reducing ability by $\mathrm{Fe}^{3+}-\mathrm{Fe}^{2+}$ transformation methods, $\mathrm{Cu}^{2+}-\mathrm{Cu}^{+}$reducing ability and phosphomolybdenum method of the compounds (2a-k) to the standard antioxidant compounds BHA and Ascorbic acid at $10 \mu \mathrm{M}$

\begin{tabular}{cccc}
\hline $\begin{array}{c}\text { Tested } \\
\text { compounds }\end{array}$ & $\begin{array}{c}\mathrm{Fe}^{3+}-\mathrm{Fe}^{2+} \text { reducing } \\
\text { ability }^{\mathrm{a}}\end{array}$ & $\begin{array}{c}\mathrm{Cu}^{2+}-\mathrm{Cu}^{+} \text {reducing } \\
\text { ability }^{\mathrm{a}}\end{array}$ & $\begin{array}{c}\text { Phosphomolybdenum } \\
\text { assay }^{\mathrm{b}}\end{array}$ \\
\hline $\begin{array}{c}\text { Ascorbic acid } \\
01\end{array}$ & 0.3490 & 0.3130 & 372.71 \\
$\mathbf{2 a}$ & 0.4013 & 0.3612 & 388.12 \\
$\mathbf{2 b}$ & 0.1272 & 0.1420 & 93.48 \\
$\mathbf{2} \mathbf{c}$ & 0.1272 & 0.1063 & 111.27 \\
$\mathbf{2 d}$ & 0.3079 & 0.2897 & 282.16 \\
$\mathbf{2 e}$ & 0.3324 & 0.3006 & 348.67 \\
$\mathbf{2 f}$ & 0.2709 & 0.2513 & 276.17 \\
$\mathbf{2 g}$ & 0.2681 & 0.2486 & 307.22 \\
$\mathbf{2 h}$ & 0.2953 & 0.1953 & 294.22 \\
$\mathbf{2} \mathbf{2}$ & 0.1536 & 0.1411 & 101.14 \\
$\mathbf{2}$ & 0.1163 & 0.0931 & 83.87 \\
$\mathbf{2} \mathbf{k}$ & 0.0615 & 0.0791 & 78.24 \\
\hline
\end{tabular}

${ }^{a}$ The values are expressed as absorbance. High absorbance indicates high reducing power.

${ }^{b}$ The values are expressed as equivalent of Ascorbic acid $(\mu \mathrm{M} / \mathrm{mg})$

\section{Cupric ion reducing ability}

All the synthesized compounds were performed cupric ion reducing ability assay ${ }^{21}$. This method utilizes the copper(II)-neocuproine reagent as the chromogenic oxidizing agent. This reagent was useful at $\mathrm{pH} 7$ and the absorbance of the $\mathrm{Cu}(\mathrm{I})$-chelate formed as a result of redox reaction with the samples was measured at $450 \mathrm{~nm}$. Among the synthesized derivatives, compounds $\mathbf{2 c}, \mathbf{2} \mathbf{b}, \mathbf{2 e}$ and $\mathbf{2 d}$ exhibited the marked cupric ion reducing ability. All other indole-2-carboxylic acid aryl-amine conjugates such as $\mathbf{2 f}$ and $\mathbf{2 a}$ showed moderate cupric reducing activity. The results are showed in (Table 3).

\section{Phosphomolybdenum method}

The antioxidant activity for the synthesized compounds (2a-k) was evaluated by using phosphomolybdate method ${ }^{22}$. It determines the total antioxidant capacity. This assay is based on the reduction of $\mathrm{Mo}(\mathrm{VI})$ to $\mathrm{Mo}(\mathrm{V})$ in presence of the antioxidant compounds and the subsequent formation of a green phosphate $\mathrm{Mo}(\mathrm{V})$ complex at acidic $\mathrm{pH}$, which is measured at $695 \mathrm{~nm}$. The antioxidant capacity of the compounds was determined for $10 \mu \mathrm{M}$ concentration. Among the synthesized analogues compound $\mathbf{2 c}, \mathbf{2 e}, \mathbf{2 f}, \mathbf{2 b}$ and $\mathbf{2 d}$ displayed good reducing ability of $\mathrm{Mo}(\mathrm{VI})$ to $\mathrm{Mo}(\mathrm{V})$ in better way due to the presence of electron donating groups such as hydroxy and methoxy in the substituted phenyl ring.

\section{Antioxidant activities}

\section{DPPH Free radical scavenging activity}

The newly synthesized compounds were screened for free radical scavenging activity by DPPH method ${ }^{18}$. Compounds of different concentrations were prepared in distilled ethanol, $1 \mathrm{~mL}$ of each compound solutions (2a-k) having different concentrations (10, 25, 50, 100, 200 and $500 \mu \mathrm{M}$ ) were taken in different test tubes, $4 \mathrm{~mL}$ of $0.1 \mathrm{mM}$ ethanol solution of DPPH was added and shaken vigorously. The test tubes were then incubated in the dark room at r.t. 
for $20 \mathrm{~min}$. A DPPH blank was prepared without the compound and ethanol was used for the baseline correction. Changes (decrease) in the absorbance at $517 \mathrm{~nm}$ were measured using a UV-visible spectrometer (Shimadzu $160 \mathrm{~A}$ ). The radical scavenging activities were expressed as the inhibition percentage and were calculated using the formula:

$$
\text { Radical scavenging activity } \left.(\%)=\left[\left(A_{c}-A_{s}\right) / A_{c}\right) \times 100\right]
$$

Where $A_{c}$ is absorbance of the control (without compound) and $A_{s}$ is absorbance of the compounds (2a-k). The radical scavenging activity of BHA and ascorbic acid was also measured and compared with that of the different synthesized compound. The compound concentration providing $50 \%$ inhibition $\left(\mathrm{IC}_{50}\right)$ was calculated from the graph of $\mathrm{RSA}$ percentage against compound concentrations.

\section{$\mathrm{ABTS}^{*+}$ radical scavenging activity}

The analysis of $\mathrm{ABTS}^{\circ+}$ radical scavenging activity was determined according to Re method $^{19}$. The ABTS ${ }^{*+}$ cation was produced by the reaction between $7 \mathrm{mM}$ ABTS in $\mathrm{H}_{2} \mathrm{O}$ and $2.45 \mathrm{mM}$ potassium persulfate, stored in the dark at room temperature for $12 \mathrm{~h}$. Before the usage, the $\mathrm{ABTS}^{\cdot+}$ solution was diluted to get an absorbance of $0.700 \pm 0.025$ at $734 \mathrm{~nm}$ with phosphate buffer $(0.1 \mathrm{M}, \mathrm{pH} 7.4)$. Then, $1 \mathrm{~mL}$ of $\mathrm{ABTS}^{\cdot+}$ solution was added to the $1.5 \mathrm{~mL}$ compounds (2a-k) solution in ethanol at different concentrations (10, 25, 50, 100, 200 and $500 \mu \mathrm{M} / \mathrm{mL}$ ). After $30 \mathrm{~min}$, the percentage inhibition at $734 \mathrm{~nm}$ was calculated for each concentration relative to a blank absorbance (ethanol).

The scavenging capability of $\mathrm{ABTS}^{\circ+}$ radical was calculated using the following equation: $\mathrm{ABTS}^{\circ+}$ scavenging effect $(\%)=\left[\left(\mathrm{A}_{\mathrm{c}}-\mathrm{A}_{\mathrm{s}}\right) / \mathrm{A}_{\mathrm{c}}\right] \times 100$

Where, $A_{\text {control }}$ is the initial concentration of the $\mathrm{ABTS}^{++}$and $\mathrm{A}_{\text {sample }}$ is the absorbance of the remaining concentration of $\mathrm{ABTS}^{*+}$ in the presence of the compounds (2a-k).

\section{Ferric ion $\left(\mathrm{Fe}^{3+}\right)$ reducing antioxidant power assay}

The reducing antioxidant power assay for the newly synthesized compounds was determined according to Oyaizu method ${ }^{20} .2 \mathrm{~mL}$ of the compounds (2a-k) having concentration $10 \mu \mathrm{M}$ were mixed with phosphate buffer $(2.5 \mathrm{ml}, 0.2 \mathrm{M}, \mathrm{pH} 6.6)$ and potassium ferric cyanide (2.5 mL, 1\%). The mixture was incubated at $50{ }^{\circ} \mathrm{C}$ for $20 \mathrm{~min}$. Later, the reaction mixture was acidified with trichloroacetic acid $(2.5 \mathrm{~mL}, 10 \%)$. After $\mathrm{FeCl}_{3}(0.5 \mathrm{~mL}, 0.1 \%)$ was added to this solution, the absorbance was measured at $700 \mathrm{~nm}$. The increased absorbance of the reaction mixture indicates an increased reducing power.

\section{Cupric ion $\left(\mathrm{Cu}^{2+}\right)$ reducing ability:}

The cupric reducing ability antioxidant assay was determined according to the literature method ${ }^{21}$. Briefly, a mixture of $\mathrm{CuCl}_{2}(1 \mathrm{~mL}, 0.01 \mathrm{M})$ solution, ethanolic neocuproine (Nc) $\left(1 \mathrm{~mL}, 7.5 \times 10^{-3} \mathrm{M}\right)$ solution and Ammonium acetate $(1 \mathrm{~mL}, 1 \mathrm{M})$ in a test tube were added to a solution of compounds (2a-k) having $10 \mu \mathrm{M}$ concentration along with $0.1 \mathrm{~mL}$ distilled water. The mixture was incubated for $30 \mathrm{~min}$. Then the absorbance was measured at $450 \mathrm{~nm}$ against reagent blank.

\section{Evaluation of antioxidant capacity by Phosphomolybdenum method}

The total antioxidant capacity was evaluated by the reported method ${ }^{22}$. An aliquot of $0.1 \mathrm{~mL}$ of compound solutions $(10 \mu \mathrm{M})$ was combined with $1 \mathrm{~mL}$ of reagent solution (0.6 M sulfuric acid, $28 \mathrm{mM}$ sodium phosphate and $4 \mathrm{mM}$ ammonium molybdate). In case of blank $0.1 \mathrm{~mL}$ of methanol was used in place of compound. The tubes were capped and incubated in a 
boiling water bath at $95{ }^{\circ} \mathrm{C}$ for $90 \mathrm{~min}$. After the samples had cooled to room temperature, the absorbance of the aqueous solution of each was measured at $695 \mathrm{~nm}$ against blank of spectrophotometer. The antioxidant capacities of the synthesized indole-2-carboxylic acid analogues (2a-k) were expressed as equivalent of ascorbic acid ( $\mu \mathrm{M} / \mathrm{mg}$ of compound).

\section{Conclusion}

In conclusion, we have synthesized a new series of indole-2-carboxylic acid conjugated with aryl amines (2a-k) and evaluated for their antioxidant power by various in vitro methods. Most of them demonstrated a broad spectrum of antioxidant activities. The simple indole-2carboxylic acid derivatives $\mathbf{2 b}, \mathbf{2 d}, \mathbf{2 e}$ and $\mathbf{2 f}$ were concluded as good antioxidant activity, whereas 2c displayed more potent derivative in all the in vitro antioxidant assays. It clearly demonstrated that the assembly of appropriate aryl-amine substituents on the $\mathrm{N}$-acylated indole-2-carboxylic acid moiety would lead to the enhancement for potent antioxidant derivatives. It implied that, compounds $\mathbf{2 c}, \mathbf{2 b}, \mathbf{2 d}, \mathbf{2 e}$ and $\mathbf{2 f}$ may be considered as new promising lead candidates for further design and synthesis of this class of antioxidants.

\section{References}

1. Halliwell B and Gutteridge J M, Free Radicals in Biology and Medicine. Clarendon Press. Oxford, 1989, 23-30.

2. Gulcin I, Buyukokuroglu M E, Oktay M and Kufrevioglu O I, J Pineal Res., 2002, 33, 167-171.

3. Gulcin I, Oktay M, Kufrevioglu O I and Aslan A, J Ethnopharmacol., 2002, 79, 325-329.

4. Aruoma O I and Halliwell B, Free Radicals and Food Additives, Taylor and Francis, London, 1991.

5. Halliwell B, Murcia M A, Chirico S and Aruoma O I, Crit Rev Food Sci Nutr., 1995, 35, 7-20.

6. Silva F A M, Borges F and Ferreira M A, J Agric Food Chem., 2001, 49, 3936-3941.

7. Fresco P, Borges F, Dinis C and Marques M P M, Med Res Rev., 2006, 26(6), 747-766.

8. Zhu Y F, Chen C and Struther R S, In Annual Reports in Medicinal Chemistry, Doherty A M, Ed., Elsevier Academic Press. Amsterdam, 2004, 39, 99-110.

9. Lednicer D, The Organic Chemistry of Drug Synthesis, Wiley, New York, 1999, 6, 124-132.

10. Saracouglu N, Top Heterocycl Chem., 2007, 11, 1-61.

11. Suzen S, Top Heterocycl Chem., 2007, 11, 145-178.

12. Gulcin J, Amino Acids, 2007, 32, 431-438.

13. You-Zhi T and Zai-Qun L, Bioorg Med Chem., 2007, 15, 1903-1913.

14. Vijay Kumar H and Nagaraja Naik, Eur J Med Chem., 2010, 45(1), 2-10.

15. Vijay Kumar H, Kishor Kumar C and Nagaraja Naik, Med Chem Res., 2011, 20, 101-108.

16. Nagaraja Naik, Vijay Kumar H and Harini S T, Eur J Chem., 2011, 2(3), 337-341.

17. Nagaraja Naik, Vijay Kumar H and Vidyashree P B, J Phar Res., 2011, 4(8), 2686-2689.

18. Blois M S, Nature, 1958, 26, 1199-1200.

19. Re R, Pellergini N, Proteggenete A, Pannala A, Yang M and Rice Evans C, Free Radic Biol Med., 1999, 26, 1231-1237.

20. $\quad$ Oyaizu M, Jpn J Nut., 1986, 44, 307-315.

21. Apak R, Guclu K, Ozyurek M and Celik S E, Microchim Acta., 2008, 160, 413-419.

22. Prieto Pineda M and Aguilar M, Anal Biochem., 1999, 269(2), 337-341. 